\title{
DESIGN AND ANALYSIS OF CAVITATING PROPELLER
}

\author{
T. MAHESH KUMAR ${ }^{1}$, RAVI KUMAR. ${ }^{2}$, K. V. MUARALI KRISHNA ${ }^{3} \&$ P. K. DASH ${ }^{4}$ \\ ${ }^{1,2}$ Assistant Professor, Department of Aeronautical Engineering, Institute of Aeronautical Engineering,
} Hyderabad, Telangana, India

${ }^{3}$ M. Tech, Department of Mechanical Engineering, Aurobindo College of Institutions, Hyderabad, Telangana, India.

${ }^{4}$ Assistant Professor, Department of Aeronautical Engineering, MLR Institute of Technology, Hyderabad, Telangana, India

This study details experiments investigating previously unrecognized surge instability on a cavitating propeller in a water tunnel. The surge instability is first explored through visual observation of the cavitation on the propeller blades and in the tip vortices. Similarities between the instability and previously documented cavitation phenomena are noted. Measurements of the radiated pressure are then obtained, and the acoustic signature of the instability is identified. The magnitudes of the fluctuating pressures are very large, presumably capable of producing severe hull vibration on a ship. The origins of the instability are explored through the separate investigation of the cavitation dynamics and the response of the water tunnel to volumetric displacement in the working section. Experiments are conducted to quantify the dynamics of the propeller cavitation. Finally, a model is developed for the complete system, incorporating both the cavitation and facility dynamics. The model predicts active system dynamics (linked to the mass flow gain factor familiar in the context of pump dynamics) and therefore potentially unstable behavior for two distinct frequency ranges, one of which appears to be responsible for the instability. In this Project, Pro-E software is used to design the propeller. An analysis is done on Ansys Software version 13.0 Ansys is an Engineering Simulation Software (computer -aided design). It is used to find the stress, bending moment and frequency modes of the given design part.
\end{abstract}

KEYWORDS: Propeller, Cavitation Study, Cavitation Unsteady, Propeller Geometry \& Analysis

Received: Mar 24, 2018; Accepted: Apr 16, 2018; Published: May 05, 2018; Paper Id.: IJMPERDJUN201839

\section{INTRODUCTION}

Today, conventional marine propellers remain the standard propulsion mechanism for surface ships and underwater vehicles. Modifications of basic propeller geometries into water jet propulsions and alternate style thrusters on underwater vehicles have not significantly changed how we determine and analyze propeller performance. We still need propellers to generate adequate thrust to propel a vessel at some design speed with some care taken in ensuring some "reasonable" propulsive efficiency. Considerations are made to match the engine's power and shaft speed, as well as the size of the vessel and the ship's operating speed, with an appropriately designed propeller. Given that the above conditions are interdependent (ship speed depends on ship size, the power required depends on desired speed, etc.) we must at least know a priori our desired operating speed for a given vessel. This chapter deals with the different approaches for designing contra-rotating propellers using lifting line theory. First, the optimum loading criteria for single propellers are presented. A review of the various methods for designing CRP is given next. Section 2.2.3 describes the numerical implementation of two design methods for CRP. A detailed description of the interaction velocities calculation procedure, which is an important part of the design of interacting components, is given. Finally, the process of determining the shape of the blade once the loading 
distribution has been calculated is explained. As mentioned, lifting line theory is the basis for propeller design since it provides the radial distribution of loading or circulation. This distribution is obtained by use of criteria for optimum efficiency or modifications of such a distribution, for example, to reduce the hub or the tip loading, avoid cavitation, high vibratory forces, and noise, etc. Betz(1919) first derived the optimum circulation, distribution criterion for propellers operating in uniform wake by using Munk's 'displacement law' that states that the total force on a lifting surface is unchanged if an element of bound circulation is displaced in a streamwise direction. This result suggested that the ultimate forms of the vortices far downstream for an optimum circulation, distribution are true helices and is expressed as

$$
\tan \beta_{i}=\tan \beta / \sqrt{l^{*}}
$$

Where ' $l *$ ' is a dimensionless constant depending on the required thrust produced by the propeller. The condition for non-uniform or wake-adapted inflow was given by Lerbs (1952) by extending the Betz's work after including the thrust deduction and the wake fractions in his computations. Lerbs' method remains one of the universally accepted procedures for establishing the radial distribution of circulation and calculating the propeller efficiency in the context of lifting line theory. Several other criteria were developed afterward, but all gave a distribution of the hydrodynamic pitch angle $\tan \beta_{i}$ of the form

$$
\tan \beta_{i}=k \cdot F(r)
$$

Where $\mathrm{k}$ is an unknown factor related to the required thrust and $\mathrm{F}$ a function depending on the optimization criterion. A different procedure for determining optimum circulation distributions has been developed by Kerwin, Coney, and Hsin (1986). Instead of deriving optimum criteria corresponding to Betz or Lerbs a numerical version of their derivation using the calculus of variations and Lagrange multipliers, but working with the unknown circulations, is used. Interestingly, both methods yield similar results and in the limit of light loading the variational optimum approaches Lerbs' optimum.

Another method based on a genetic algorithm has been presented by Caponnetto et al. (1997). This method is based on a trial and error procedure through which several different propellers, all satisfying the design requirements are sequentially analyzed and their efficiency is calculated. Each time a more efficient propeller is found and it is used as a base for a new candidate optimum propeller, modified by a small random perturbation. Thus, neither a closed analytical form for describing efficiency nor an optimality criterion is required, even though several thousand iterations would be necessary in order to obtain 'smooth' propeller geometry. The increase of the propulsive efficiency of ships has always been the objective of naval architects and propeller designers. Several propulsor configurations have been developed aiming at improving the propulsive efficiency. Contra-rotating propellers are one of the most attractive propulsive devices, which can be expected to improve the open water efficiency remarkably by reducing the rotational kinetic energy losses. Despite the hydrodynamic advantages and the possible improvement of the propulsive efficiency that the CRP concept could offer, application to ships has been limited. A reasonable explanation can be given by considering the mechanical complexity, the increased installation cost and the high maintenance requirements associated with the installation of this 'unconventional' propulsor configuration in mechanically driven ships. The development of the podded propulsion during the past few years has removed the need for complex transmission systems and has brought the concept of CRP back into the daylight. In addition, advances in the field of electric propulsion are expected to further increase the application of CRP systems. propeller design and analysis methods, capable of treating CRP receive increased attention. 
In spite of the development of elaborate lifting surface methods, as well as the introduction of surface panel methods, lifting line theory still plays an essential role in propeller design and particularly in the preliminary design stage. According to Kerwin (1986), the Hydrodynamic design of a propeller can be thought to consist of two steps:

Establishment of the radial and chordwise distribution of circulation over the blades which will produce the desired thrust

Determination of the blade shape that will produce the prescribed circulation distribution

However, within the limitations of lifting line theory, only the radial distribution of circulation can be obtained since the blades are modeled by straight lines carrying point vortex elements. The radial circulation values are distributed chordwise by assuming that the propeller blade consists of hydrofoil sections with specific camber and thickness distributions. In this work, the focus is placed on determining optimum radial circulation distributions for CRP by presenting two numerical methods in the context of lifting line theory. Both methods were coded in MATLAB®, a widely used high-performance language for technical computing. The computer codes borrowed from the vortex-lattice lifting line formulation, utilized in the open-source propeller design code 'OpenProp' (R. W. Kimball et al. 2008). OpenProp evolved from the MIT Propeller Vortex Lattice Lifting Line Program (PVL) developed by Kerwin (2001), which was later translated into the GUI-based MATLAB® program called MPVL (Chung 2007). The ultimate goal of this work is the extension of the OpenProp's capabilities so that it can design contra-rotating propeller sets and perform a basic cavitation analysis. Before discussing the CRP design theory and it detailed numerical implementation, it is necessary to describe the vortex lattice lifting line theory as applied to the case of the single propeller design.

Unlike the actuator disc a propeller requires that the power be input into the fluid via a shaft and thus must apply a torque to the fluid. To balance this torque the flow must contain tangential velocity, or swirl, to counteract this torque. The kinetic energy in the swirl velocity, if not recovered by a stator or downstream blade row, is lost. Therefore the efficiency of an ideal propeller will be less than an actuator disc efficiency. For a propeller in uniform flow this ideal efficiency can be computed given the advance coefficient, J, and the thrust coefficient, CT. The graphical chart of these results is depicted in the Kramer diagram, which also has corrected for losses due to finite blade number. This chart does NOT include viscous losses and gives the maximum achievable efficiency of a real propeller in uniform inflow.

\section{To Use Kramer's Diagram}

- Need to Know: $\mathrm{T}$ = thrust to propel the ship (need to know ship's total resistance)

- Find CT based on your chosen propeller area.

- Choose the number of blades

- Know VA, D, N

- Use chart:

Start with $\lambda=$ Absolute advance coefficient $(\lambda=\mathrm{Va} /(\pi \mathrm{nD})=\mathrm{J} / \pi)$

Follow up the diagonal line until you hit the horizontal line that corresponds to your number of blades.

Next, go DIRECTLY up to the next horizontal line that corresponds to your known value of $\mathrm{Ct}$ which you 
calculated earlier.

Then choose the closest efficiency curve or interpolate between curves for $\eta$.

\section{Propeller Cavitation}

Cavitation occurs when the local absolute pressure is less than local vapor pressure of the fluid medium.

Cavitation Number (Inflow Velocity Based) is defined as:

$\sigma v=2(P-P v a p) / \rho A V a 2$

Sometimes Propeller Cavitation Number is defined based on Propeller tip Speed:

$\sigma \mathrm{ND}=(\mathrm{P}-\mathrm{Pvap}) /(\boldsymbol{\rho}$ N2 D2 $)$

The Critical measurement for cavitation performance is the cavitation inception point which is the conditions (i.e. Cavitation number) for which cavitation is first observed anywhere on the propeller.

Typically the Inception cavitation number as a function of Kt or J is plotted for a given propeller which defines the cavitation free operation's boundary. This boundary, typically has a bucket shape which is referred to as the cavitation bucket.

\section{CAVITATION ANALYSIS}

\subsection{Steady Cavitation in Uniform Wake}

When the propeller is assumed to operate in a uniform axial wake (open water conditions) the propeller blades experience steady flow conditions as they rotate about the propeller axis. The design of the blades is then performed by requiring a shock-free entry, a condition which is satisfied around the propeller disk given that the flow is considered to be independent of the angular position. The occurrence of cavitation ,in this case, is exclusively due to high blade loadings and can be observed in the mid-chord neighborhood on the suction side, where the pressure attains its minimum value. On the other hand, no cavitation occurs near the leading edge, since the pressure distribution on the blade sections does not exhibit local peaks near this area. Figure 2-1 shows the cavitation patterns on the blades of a free-running conventional propeller, the characteristics of which are given in Table 2-1.

Table 2.1: SR Propeller Characteristics used in Steady Cavitation Analysis

\begin{tabular}{|l|l|}
\hline Number of blades & $\mathrm{Z}=3$ \\
\hline Blade Diameter & $2.5 \mathrm{~m}$ \\
\hline Hub Diameter & $0.5 \mathrm{~m}$ \\
\hline Thrust Loading & $\mathrm{Ct}=0.932$ \\
\hline Advance coefficient & $\mathrm{Js}=0.738$ \\
\hline Shaft Centerline Depth & $\mathrm{H}=2 \mathrm{~m}$ \\
\hline
\end{tabular}

The particulars of the propeller have been selected such that cavitation would occur and its patterns on the blade's surface could be observed. Comparing the results between VLM and XFOIL, the former method yields smaller cavitating areas (34.3\% compared to $40.6 \%$ ). The percentage that appears on the 3D color plots corresponds to the blade positioned at the 12 o'clock location. Areas, where the local pressure is lower than the vapor pressure, are marked in red, whereas the color for the rest of the surface corresponds to the local pressure coefficient. 


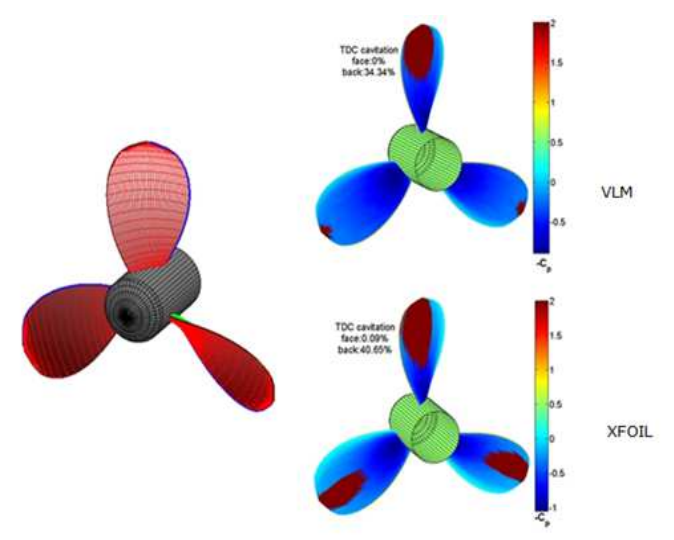

Figure 2.1.1: Geometry and Steady Cavitation Patterns of a Conventional Propellor

Under the same conditions (see Table 4-1) was also examined using VLM. In this case, though, the total expanded area ratio is larger by $33 \%$ compared to the conventional propeller. Figure 4-2shows that cavitation will be significantly decreased on the forward propeller. The fact that the aft propeller blades will not develop cavitation at all is explained by the reduced circulation values compared to the forward one, which leads to aft blade sections having lower lift coefficients.

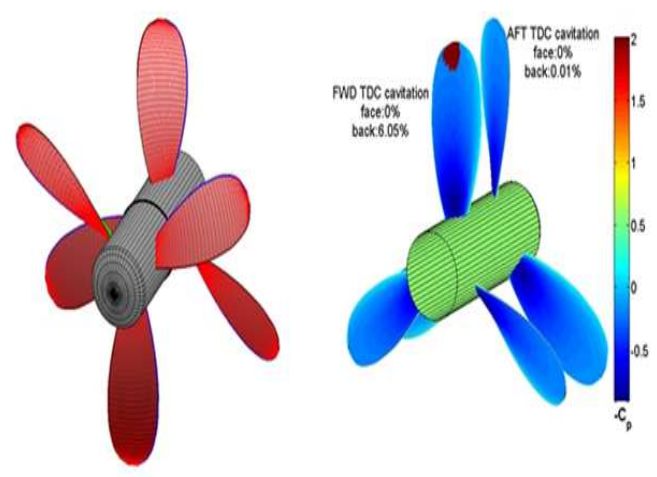

Figure 2.1.2: Geometry and Steady Cavitation Pattern of a CRP Set Using VLM

\subsection{Unsteady Cavitation}

In the previous section operation under open-water conditions, in which the propellers advanced into the undisturbed water, was assumed. In practice, however, propellers are placed behind a ships hull where the inflow conditions are far from uniform. Flow measurements at the actual propeller locations yield wakes that vary both temporally and spatially. Even if the temporal variation is ignored, a more realistic representation of the wakefield could be obtained by considering that it is a function of the radius and the angular position in the plane of the propeller disk. Figure 2.2.1 shows the geometry and the cavitation analysis results in a conventional single propeller operating in the non-uniform axilal wake presented earlier. The particulars of the propeller are given in Table 2.2.1. The advance coefficient is the one yielding the maximum propeller efficiency for the specific thrust loading. 
Table 2.2.1: SR Propeller Characteristics Used in Unsteady Cavitation Analysis

\begin{tabular}{|l|c|}
\hline Number of blades & $\mathrm{Z}=4$ \\
\hline Blade Diameter & $2 \mathrm{~m}$ \\
\hline Hub Diameter & $0.4 \mathrm{~m}$ \\
\hline Thrust Loading & $\mathrm{Ct}=0.69$ \\
\hline Advance coefficient & $\mathrm{Js}=0.89$ \\
\hline Shaft Centerline Depth & $\mathrm{H}=2 \mathrm{~m}$ \\
\hline
\end{tabular}

It is evident from Figure 4-5 that cavitation occurs only on the blade oriented at the 12 o'clock position. The axial velocity in that region is lower than the mean axial velocity value, for all radial locations, and the blade sections operate at extremely high (and positive) angles of attack.

Moreover, the cavitation numbers in that sector are lower due to the decreased submergence depths. Thus, areas, where back sheet cavitation occurs near the leading edge on the suction side, are formed. Comparing this pattern to the one developed due to steady cavitation (Figure 4-1), we conclude that not only highly loaded propellers are prone to cavitation inception, but also adverse flow conditions, such as operation in a highly non-uniform wakefield, can lead to the onset of cavitation. In order to decrease the severity of the phenomenon, thicker blade sections should be adopted, given the cavitation sensitivity of thin sections.
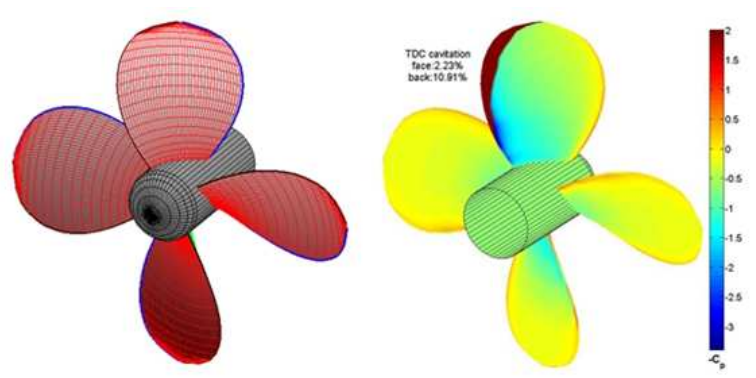

Figure 2.2.1: Geometry and Unsteady Pattern of Conventional Patterns using VLM

A contra-rotating propeller set was subjected in the same non-axisymmetric wakefield. The underlying assumption was that the wake is the same for the forward and the aft components of the CRP, even though in reality, at the aft propeller plane the axial velocity field will probably be more uniform. The geometry and the cavitation patterns on the blades of the forward and aft screws are shown in Figure 2.2.2
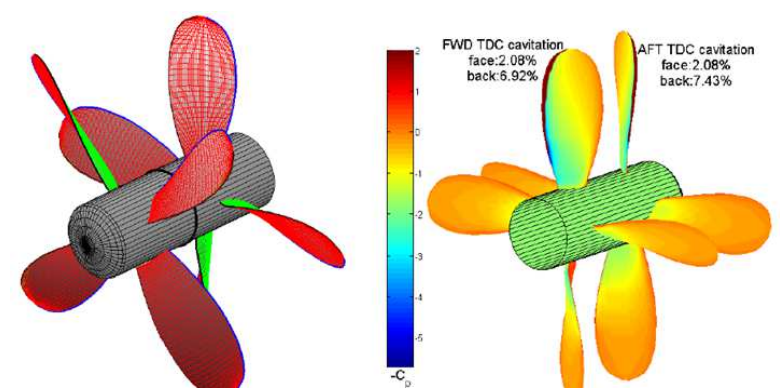

Figure 2.2.2: Geometry and Unsteady Patterns of CRP Set using VLM

The CRP set was required to develop the same thrust and had the same diameter and expanded area ratio as the single propeller. However, a higher advance coefficient was assumed which effectively means that each component of the 
set rotated slower. This selection is supported by the results of the parametric design study presented in the first part of this thesis. According to this, the optimal advance coefficient for CRPs in terms of efficiency is lower than the one for single rotation propellers. Thus, the small improvement in the cavitation behavior of the CRP set observed in Figure 6-6 can be attributed to the higher values of the cavitation index, as a consequence of the lower rotational speeds. Nevertheless, this improvement alone could not justify the selection of a contra-rotating propeller over a conventional one, given the increased mechanical complexity and cost of the former. It is the combined improvement in hydrodynamic efficiency and cavitation performance that makes the CRP set a very attractive choice. Table 2.2.3 presents the results of the two propulsor variants designed for operation in the non axisym-metric axial wake field.

Table 2.2.3: Comparison between SRP - CRP Design for Operation in a Non-Uniform Axial Wake

\begin{tabular}{|l|c|c|}
\hline & SRP & CRP \\
\hline Advance coefficient (Js) & 0.89 & 1.36 \\
\hline Rotational speed (N) & 168.5 RPM & 110 RPM \\
\hline Suction Side(s) cavitation & $10.9 \%$ & $7.2 \%$ \\
\hline Efficiency (n) & $66.2 \%$ & $74.4 \%$ \\
\hline
\end{tabular}

Apart from the smaller cavitation patterns, the CRP pair achieves an improvement in the hydrodynamic efficiency of $12.4 \%$ over the conventional single propeller.

\section{METHODOLOGY}

\subsection{Finite Element Modeling of the Propeller}

Modeling of the propeller is done using CATIA V5 R 17. In order to model the blade, it is necessary to have sections of the propeller at various radii. These sections are drawn and rotated through their respective pitch angles. Then all rotated sections are projected onto right circular cylinders of respective radii as shown in the figure below. Now by using multi section surface options, the blade is modeled.

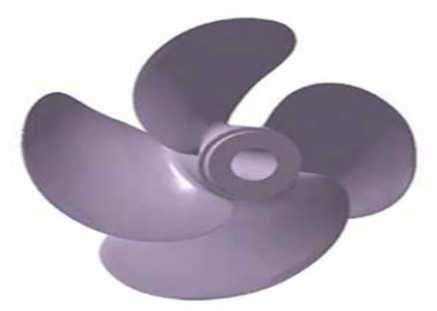

Figure 3.1.1: Final Solid Model of Propeller

\subsection{Mesh Generation using Hyper mesh}

The solid model is imported to HYPERMESH 10.0 and hexahedral mesh is generated for the same. The meshed model is shown in figure 5.2. The meshing was done by splitting it into different areas and the 2D mapped mesh was done and then it was converted into a 3D mesh using the tool linear solid. The number of elements created is 27,388 and the number of nodes created is 52,412. Quality checks are verified for the meshed model. Jacobian, warpage and aspect ratio are within permissible limits. Now the pressures are applied to the propeller which is obtained in FLUENT 6.3. Then the meshed model is imported into the ANSYS 


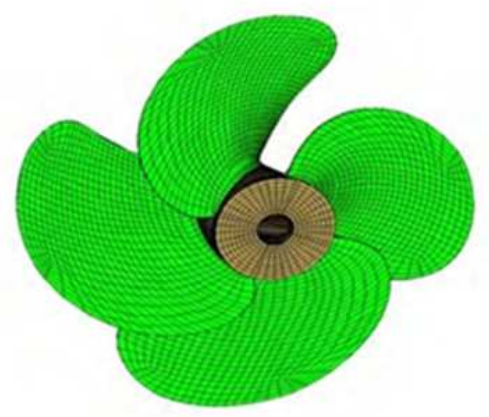

Figure 3.2.1: Aluminium Propeller Meshed Model

For meshing the composite propeller the solid model is imported into hyper mesh and the elements are selected for various lay-up sequences as given by their thickness. Assure that proper connectivity exists between the layers. Now again the pressures are applied to the propeller which is obtained in FLUENT 6.3. Then the meshed model is imported into the ANSYS.

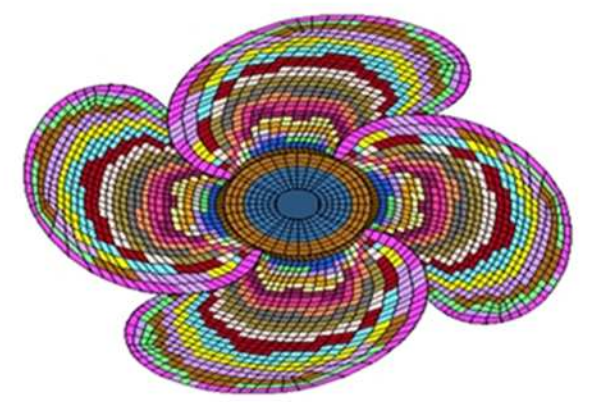

Figure 3.2.2: Composite Propeller Meshed Model

\title{
3.3. Exporting Mesh to the ANSYS 11.0
}

First, delete all the surfaces and $2 \mathrm{~d}$ elements before exporting to the ANSYS so that only 3D elements are exported. Now select the user profile in the preferences, then go to utility menu and mention the ET type as solid45 if it is aluminium or solid 46 for composite material, specify material properties and real constants if necessary. Then go to a 3D option and mention the ET type and element types. Update all the components in the component option and at last renumber all the components. Now go to export option to export the FE model to the ANSYS.

\subsection{Material Properties of Propeller}

\author{
Aluminum properties \\ Young's modulus $\mathrm{E}=70000 \mathrm{MPa}$ \\ Poisson's ratio $\mu x y=0.34$ \\ Mass density $\quad=2700 \mathrm{~kg} / \mathrm{m} 3$ \\ Damping co-efficient $=0.03$
}


Table 3.4.1: Material Properties for Composite Propeller used for Present Work

\begin{tabular}{|l|l|}
\hline Mat no 1: S2Glass Fabric/Epoxy & Mat no 2: Carbon UD/Epoxy \\
\hline $\mathrm{Ex}=22.925 \mathrm{Gpa}$ & $\mathrm{Ex}=120 \mathrm{Gpa}$ \\
\hline $\mathrm{Ey}=22.925 \mathrm{Gpa}$ & $\mathrm{Ey}=10 \mathrm{Gpa}$ \\
\hline $\mathrm{Ez}=12.4 \mathrm{Gpa}$ & $\mathrm{Ez}=10 \mathrm{Gpa}$ \\
\hline$\mu_{\mathrm{xy}}=0.12$ & $\mu_{\mathrm{xy}}=0.16$ \\
\hline$\mu_{\mathrm{yz}}=0.2$ & $\mu_{\mathrm{yz}}=0.2$ \\
\hline$\mu_{\mathrm{xz}}=0.2$ & $\mu_{\mathrm{xz}}=0.16$ \\
\hline $\mathrm{Gxy}=4.7 \mathrm{Gpa}$ & $\mathrm{Gxy}=5.2 \mathrm{Gpa}$ \\
\hline $\mathrm{Gyz}=4.2 \mathrm{Gpa}$ & $\mathrm{Gyz}=3.8 \mathrm{Gpa}$ \\
\hline $\mathrm{Gzx}=4.2 \mathrm{Gpa}$ & $\mathrm{Gzx}=6 \mathrm{Gpa}$ \\
\hline$\rho=1.8 \mathrm{gm} / \mathrm{cc}$ & $\rho=1.6 \mathrm{gm} / \mathrm{cc}$ \\
\hline
\end{tabular}

\subsection{Boundary Conditions and Loads}

The propeller is fixed at the hub and shaft intersections so all DOF conditions apply to the nodes. Pressure is applied to the total propeller which is obtained from the CFD analysis.

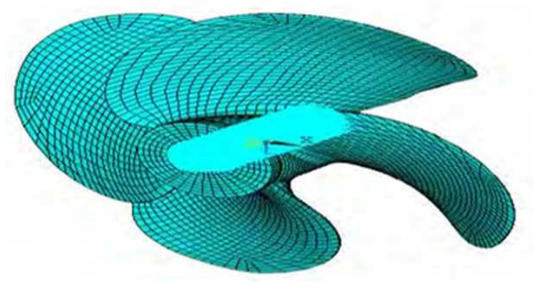

Figure 3.5.1: Propeller FE Model in ANSYS

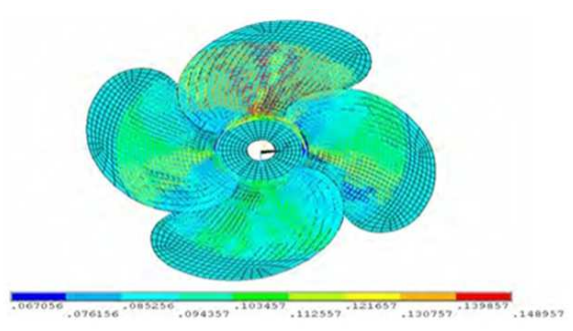

Figure 3.5.2: Propeller FE Model in ANSYS Pressures

\section{RESULTS}

Propeller made of Aluminum and another one made of composite material is chosen for FE analysis. At present the results of stress analysis are presented in the form of figures and graphs by selecting the effective software like ANSYS.

\subsection{Stress Analysis of Aluminum Propeller}

The deformation of aluminum propeller is shown in figure 6-1 and the maximum deflection was found as $0.038611 \mathrm{~mm}$. The stresses and displacements in the $\mathrm{x}, \mathrm{y}$ and $\mathrm{z}$ directions are shown in table 4.1.1. 
Table 4.1.1: Static Analysis Results of Aluminium Propeller

\begin{tabular}{|l|c|}
\hline \multicolumn{1}{|c|}{ Aluminium Propeller } & Results \\
\hline Deflection & $0.038611 \mathrm{~mm}$ \\
\hline Max. Normal stress & $9.011 \mathrm{~N} / \mathrm{mm} 2$ \\
\hline First principal stress & $12.405 \mathrm{~N} / \mathrm{mm} 2$ \\
\hline Second principal stress & $4.849 \mathrm{~N} / \mathrm{mm} 2$ \\
\hline Third principal stress & $2.258 \mathrm{~N} / \mathrm{mm} 2$ \\
\hline
\end{tabular}

Table 4.1.2: Maximum Stress at the Root Section

\begin{tabular}{|c|c|}
\hline FROM ANSYS & FROM BURILL METHOD \\
\hline $9.011 \mathrm{MPa}$ & $8.9 \mathrm{MPa}$ \\
\hline
\end{tabular}

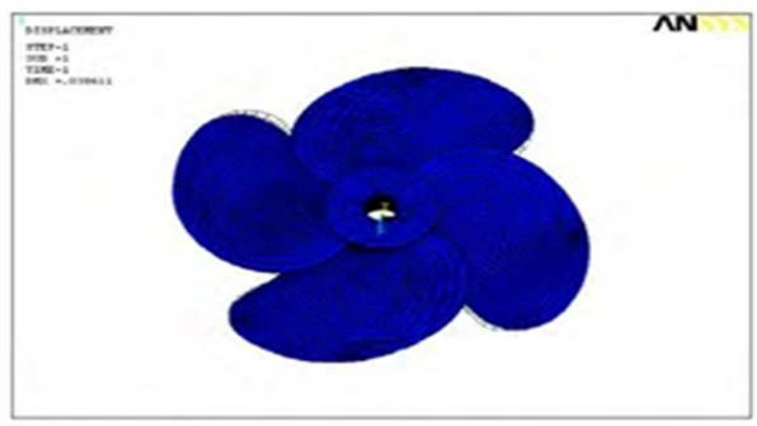

Figure 4.1.1: Deformation of Aluminium Propeller

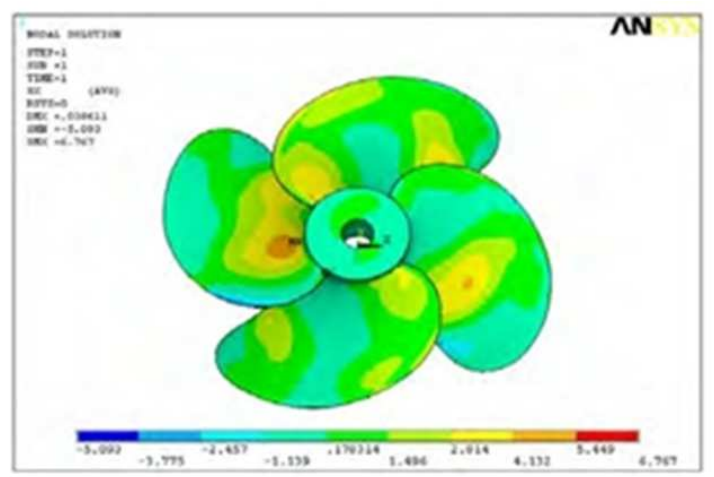

Figure 4.1.2: Stress of Aluminium Propeller in X-Direction

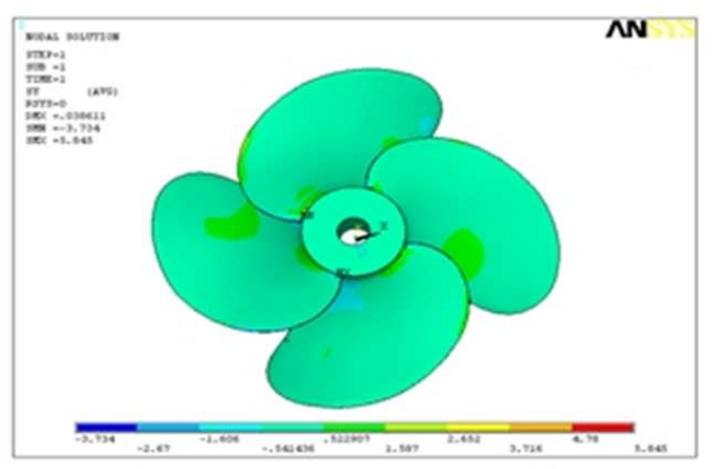

Figure 4.1.3: Stress of Aluminium Propeller in Y-Direction 


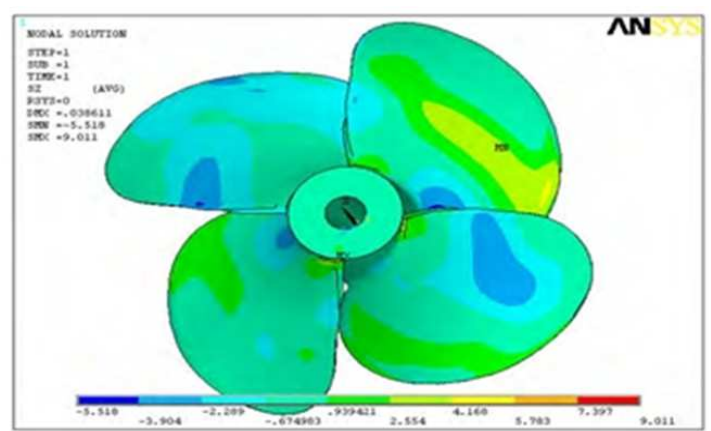

Figure 4.1.4: Stress of Aluminium Propeller in Z-Direction

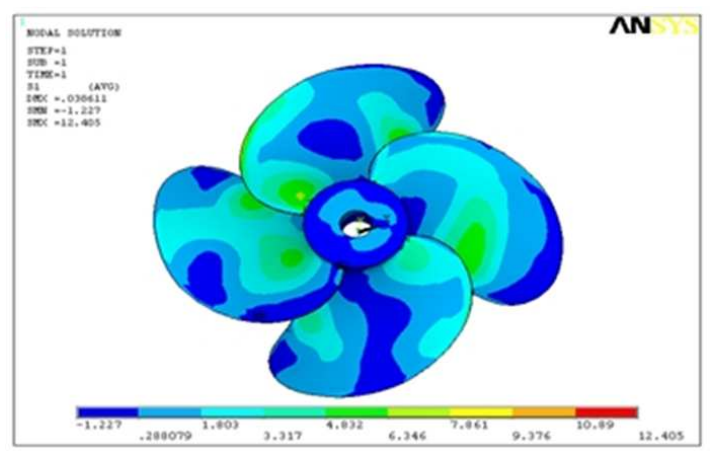

Figure: 4.1.5: 1st Principal Stress of Aluminium Propeller

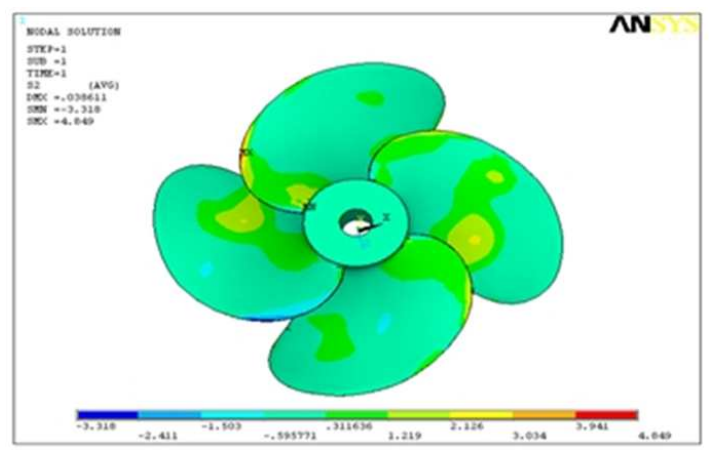

Figure 4.1.6: $2^{\text {nd }}$ Principal Stress of Aluminium Propeller

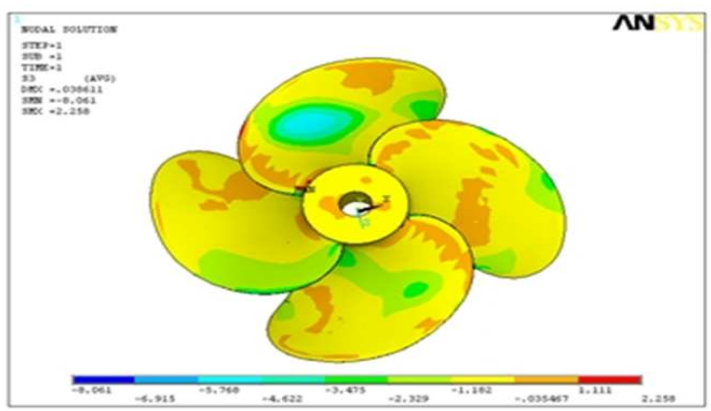

Figure 4.1.7: $3^{\text {rd }}$ Principal Stress of Aluminium Propeller

4.2. Stress Analysis of Composite Propeller

Stress analysis of composite propeller is carried out so as to check the bonding strength between various layers of the composite propeller and Inter-laminar shear stresses are calculated. 
Maximum deflection for the composite propeller with 40 layers was found to be $0.27504 \mathrm{~mm}$ as shown in figure 6-8. The maximum normal stress was found to be $7.995 \mathrm{~N} / \mathrm{mm} 2$ in the $\mathrm{x}$-direction as shown in figure 6-9. Table 3 shows induced deformations and stresses in a composite propeller.

The deflection for composite propeller was found to be around $0.27504 \mathrm{~mm} \mathrm{~mm}$ for all layers which are more than that of Aluminum propeller i.e. $0.038611 \mathrm{~mm}$. Interlaminar shear stresses were calculated for the composite propeller for 40 layers and the maximum inter-laminar shear stress is found on the layer 40 .

\section{Table 3.2.1: Stress Analysis Results of Composite Propeller}

\begin{tabular}{|l|c|}
\hline \multicolumn{1}{|c|}{ Composite Propeller } & Results \\
\hline Max deflection & $0.27504 \mathrm{~mm}$ \\
\hline Max. normal stress & $7.992 / \mathrm{mm} 2$ \\
\hline Maximum Inter-laminar shear stress & $1.353 \mathrm{~N} / \mathrm{mm} 2$ \\
\hline
\end{tabular}

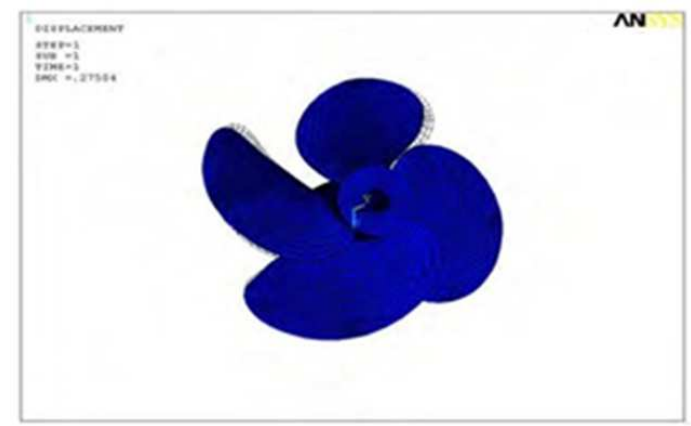

Figure 4.2.1: Deformation of Composite Propeller

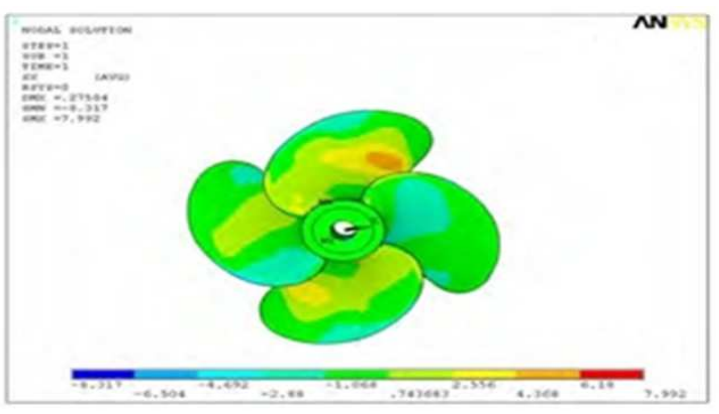

Figure: 4.2.2 Stress of Composite Propeller in X-Direction

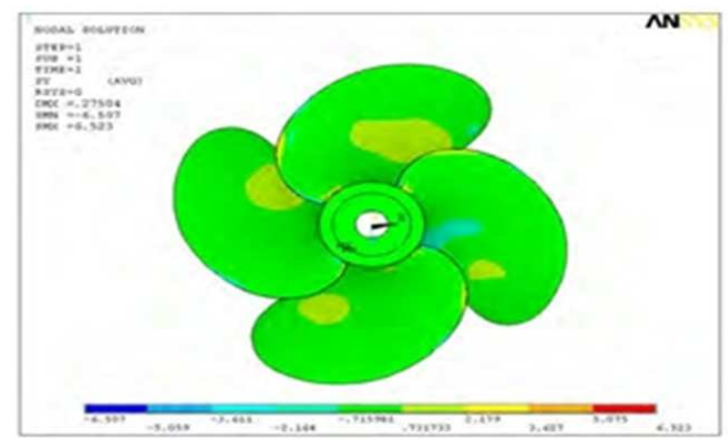

Figure 4.2.3: Stress of Composite Propeller in Y-Direction 


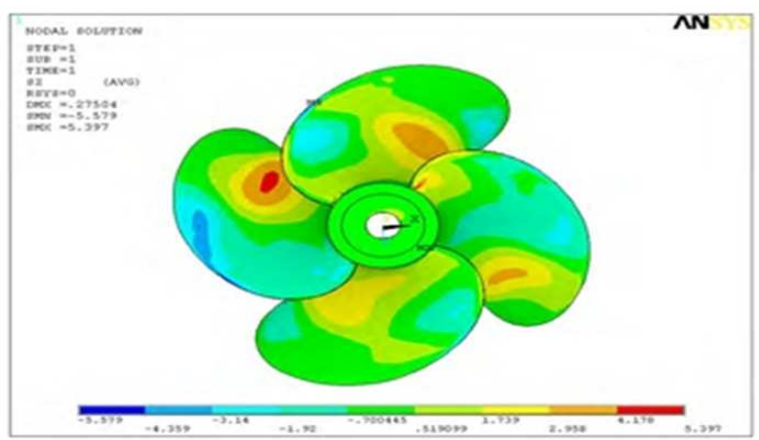

Figure: 4.2.4 Stress of Composite Propeller in Z-Direction

\section{DISCUSSIONS}

Throughout this thesis contraction of the slipstream was not considered. Even though this might not have a significant effect on the design of single propellers, it plays an important role for CRR where accurate predictions for the interaction velocity fields are required. The exclusion of the wake contraction makes the computation of the interaction velocities much easier. If this effect were to be taken into account, tracking of contracting streamlines would be necessary, something that could be approached in the following ways: Assuming that the actual wake contraction occurs over a short distance, compared to the propeller diameter, a better approximation of the wake geometry would be to model it as purely helical but contracted to some new radius. Empirical contraction ratios based on water tunnel measurements indicate that this ratio is close to 0.83 (J. E. Kerwin \& Lee 1978)

Instead of using empirical corrections, analytical solutions for the contraction ratios and the trajectories of the contracting streamlines could be implemented. Thus, the effects of the actual clearance between the propulsor components could be predicted accurately. Closed form solutions for the velocities and the stream functions for a wide range of thrust loadings have been given by Conway $(1995 ; 1998)$. Moreover, in the current methodology, the selection of the chord lengths is not part of the propeller optimization process, but it is a direct user input. The optimization procedure could be greatly improved by taking into account the effects of the chord length on the viscous drag, the cavitation performance and the structural strength of the blades, such that the selection of chord lengths could be made part of the circulation optimization scheme.

All of the above points requiring further investigation refer to the design problem, where the geometric pitch and the loading of the blades are of interest. The inverse design problem, in which the thrust and the torque coefficients at off design points are sought, given the blade geometry, should also be addressed. A procedure for obtaining the performance curves for single propellers using lifting line theory has been developed by Epps (2010) and could be extended to treat CRP as well. Validation using experimental data and inclusion of lifting surface corrections would result in a complete and reliable preliminary design and analysis tool.

\section{CONCLUSIONS}

The following conclusions are drawn from the present work

- The stresses of the composite propeller obtained in a static analysis are within the allowable stress limit.

- The maximum deflection for the aluminum propeller is $0.038611 \mathrm{~mm}$ and the maximum deflection for the composite propeller is $0.275 \mathrm{~mm}$. 
- The maximum normal stress for the aluminum propeller is $9.011 \mathrm{~N} / \mathrm{mm} 2$ and the maximum normal stress for the composite propeller is $7.99 \mathrm{~N} / \mathrm{mm} 2$, from analytical method $8.9 \mathrm{MPa}$.

- The first, second and third principle stresses for aluminum propeller are $12.405 \mathrm{~N} / \mathrm{mm} 2,9.849 \mathrm{~N} / \mathrm{mm} 2$, and 2.258 $\mathrm{N} / \mathrm{mm} 2$.

- The stress of a composite propeller in X, Y and Z directions are $7.992 \mathrm{~N} / \mathrm{mm} 2,6.523 \mathrm{~N} / \mathrm{mm} 2$, and 5.397 N/mm2.

\section{REFERENCES}

1. J. F. Tsai, H. J. L. a., Analysis of Underwater Free Vibrations of a Composite Propeller Blade. Journal of REINFORCED PLASTICS AND COMPOSITES, 2008. Vol. 27, No. 5: p. 447-458.

2. H. J. Lin, W. M. L. a. Y. M. K., effect of stacking sequence om nonlinear hydroelastic behavior composite propeller Journal of Mechanics, 2010. Vol. 26, No. 3: p. 293-298.

3. Toshio Yamatogi, H. M., Kiyoshi Uzawa, Kazuro Kageya, Study on Cavitation Erosion of Composite Materials for Marine Propeller. 2010.

4. Young, Y. L., Fluid-structure interaction analysis of flexible composite marine propellers. Journal of Fluids and Structures, 2008. 24(6): p.799-818

5. Taylor, D. W, “The speed and power of ships", Washington, 1933.

6. J. E. Conolly, "Strength of Propellers", reads in London at a meeting of the royal intuition of naval architects on Dec 1, 1960 , pp 139-160.

7. Krishnakanth, P. V., et al. "Structural \& thermal analysis of gas turbine blade by using FEM." International Journal of Scientific Research Engineering \& Technology (IJSRET) 2.2 (2013): 060-065.

8. Chang-sup lee, yong-jik kim, gun-do kim and in-sik nho. "Case Study on the Structural Failure of Marine Propeller Blades" Aeronautical Journal, Jan 1972, pp87-98

9. G. H. M. Beek, visitor, Lips B. V., Drunen. "Hub-Blade Interaction In PropellerStrength", the society of naval architects andmarine engineers, May 24-25, 1978, pp 19-1-19-14.

10. W. J. Colclough and J. G. Russel. "The Development of a Composite Propeller Blade with a CFRP Spar" Aeronautical Journal, Jan 1972, pp53-57.

11. Gau-Feng Lin "Three Dimensional Stress Analysis of a Fiber Reinforced Composite Thruster Blade”, the society of naval architects and marine engineers, 1991.

12. J. P Ghosh and R. P Gokaran. "Basic ship propulsion”, 2004. 